\title{
Performance assessment of an energy harvesting system located on a copter
}

\author{
A. Koszewnik ${ }^{\mathrm{a}}$ and D. Oldziej ${ }^{\mathrm{b}}$ \\ Department of Automatics Control and Robotics, Bialystok University of Technology, \\ Wiejska Street 45C, 15-351 Bialystok, Poland
}

Received 21 August 2018 / Received in final form 18 December 2018 Published online 23 August 2019

\begin{abstract}
Energy harvesting from moving structures for autonomous electrically powered applications, i.e. unmanned aerial vehicles (UAVs), especially in the case quadrotors, has been a common subject of scientific investigations in last years. Most of them have looked into improving the endurance of electrically powered UAVs using technologies such as photovoltaic or vibration harvesting. UAV platforms have a limited amount of power for electrical suppling. In order to keep a constant time of flight duration, the UAV can be equipped with an additional battery, which increases the total mass and causes higher energy losses. Therefore, we address this problem in our paper by proposing a vibration-based energy harvesting system. It consists of piezoelectric harvesters integrated into a frame of BLDC rotors. In order to check efficiency of the energy harvesting system, the copter is equipped with MFC harvesters. Taking into account this solution, the distributedparameters of the electromechanical system in the modal coordinates are estimated using numerical methods. The simulation results showedhow the duty cycle of the PWM signal applied to the BLDC rotor and resistive load connected to the system influence on voltage generating by the piezo harvester, while experimental test allow verify them.
\end{abstract}

\section{Introduction}

Energy harvesting from moving structures for autonomous electrically powered applications, i.e. unmanned aerial vehicles (UAVs), especially in the case of multiple motors like a quadrocopter, is a common subject of scientific in vestigationin last years. Most of them have looked into improving the endurance of electrically powered UAVs using energy harvesting technologies such as photovoltaic harvesting and vibration harvesting $[1,2]$.

Among various types of energy harvester equipment, a piezoelectric material is one effective component to convert the surrounding mechanical vibration energy into the electrical energy directly [3-5]. From prior literature during the last two decades, the piezoelectric material have long been used as sensors or actuators [6]. Although the mechanical vibration takes place in most of the structures, the possible harvested

a e-mail: a.koszewnik@pb.edu.pl

b e-mail: d.oldziej@pb.edu.pl 
a)

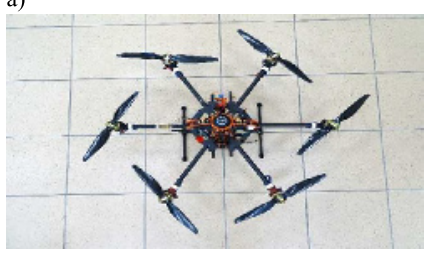

b)

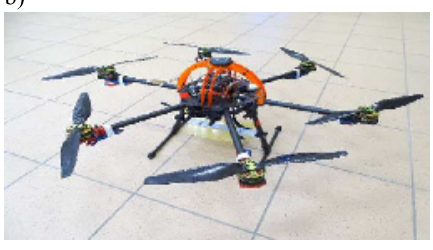

Fig. 1. The six-rotor copter made by the co-author in Bialystok University of Technology.

performance of the piezoelectric is not high and the energy level is low in micro- or milli-watt orders [7]. However, with some improvement on the piezoelectric materials and their fabrication process, as well as low energy consumption of the electronic circuit design makes a possibility of using piezoelectric elements in a wider range of applications. One particular area where piezoelectric energy harvesting proves to be useful is in scavenging vibration energy during UAVs flights. It is especially shown in papers published by Anton, who was the first to design and investigate novel piezoelectric devices installed on UAV platforms [8]. For instance, the author in reference [9] showed the piezoelectric patch with a thin-film battery as a multifunctional self-charging device for scavenging energy. In other paper the same author with Leo presented a hybrid device, containing piezo-electric stripes, macro-fiber andpiezo-fiber composites, that allows to harvest energy from wing vibrations [9]. As a result, further development of these systems lead to using the proposed solutions for platforms with many rotors having more benefits than a classical fixed wing [10,11]. Taking into account their features such as a large amount of controllability, hovering and maneuverability, it can be assumed that they will need more energy. Therefore, the UAV platforms were retrofitted with an additional battery or a battery with higher current efficiency. As a result, the maneuverability of the UAV increased, but at the expense of the flight duration time.

In this paper, the vibration-based energy harvesting system is proposed for the commonly using system shown in Figure 1, but with other approach to prolong the duration of the flight time. The considered system consists of a piezoelectric harvester integrated into a single arm of the UAV frame with BLDC rotors is considered as a smart cantilever beam with tip mass. In order to provide high efficiency of the energy harvesting system, the six-copter was equipped with a macro fiber composites (MFC) harvester of a high compliance. Taking into account the proposed solution, the distributed-parameters of the electromechanical system in the modal coordinates are assessed based on the analytical approach and using numerical methods. Then, experimental investigations performed on the lab stand allowed to verify the obtained simulation results and determine the equivalent circuit model used in the energy harvesting systems.

\section{Electromechanical model of a single arm of the UAV frame with energy piezo-harvester - analytical approach}

In this section a brief description of electromechanical model distributed-parameters of a single arm of the considered UAV platformwith BLDC rotor is analyzed. The carbon-fiber cantilever pipe representing the host structure is equipped with one piezo-patch harvester PPA-1001 located on top of the frame by using overlay. As a result, a smart structure, shown in Figure 2, is obtained. Parameters of the arm and the piezo-harvester adjusted to its structure are collected in Table 1. 
a)

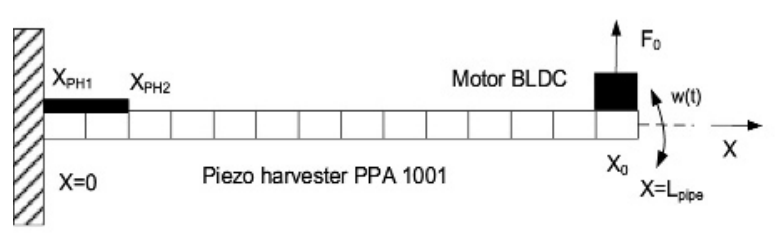

b)

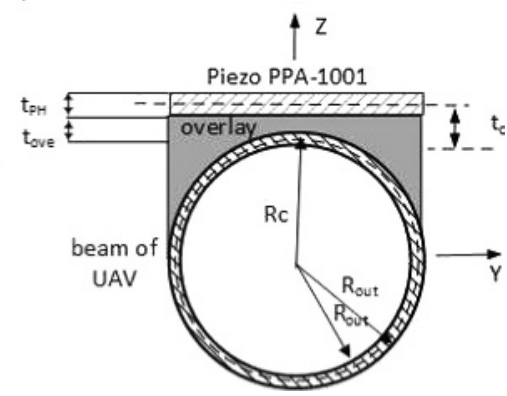

Fig. 2. The cantilever steel beam with a piezo-actuator and a piezo-harvester: (a) the model, (b) cross section with consideration of neutral axes of the beam and the piezo-harvester.

Table 1. Parameters of the host structure and the piezo-element [14].

\begin{tabular}{|c|c|c|c|c|c|c|c|c|}
\hline \multirow[t]{2}{*}{ Parameter } & \multicolumn{2}{|c|}{$\begin{array}{l}\text { Tip mass } \\
\text { (rotor) }\end{array}$} & \multicolumn{4}{|c|}{$\begin{array}{l}\text { Cantilever beam } \\
\text { (Pipe }+ \text { rotor stand housing) }\end{array}$} & \multicolumn{2}{|c|}{$\begin{array}{l}\text { Piezo- } \\
\text { harvester } \\
\text { PPA-1001 }\end{array}$} \\
\hline & \multicolumn{2}{|c|}{ Aluminum } & \multicolumn{2}{|c|}{ Carbon-fiber } & \multicolumn{2}{|c|}{ Carbon-fiber } & \multicolumn{2}{|c|}{$\begin{array}{l}\text { PZT-5H } \\
\text { (the main } \\
\text { layer material } \\
\text { of PPA-1001) }\end{array}$} \\
\hline Radius (m) & $R_{\text {tip }}$ & 0.0235 & $\begin{array}{l}R_{\text {out }} \\
R_{\text {in }}\end{array}$ & $\begin{array}{l}0.0080 \\
0.0065\end{array}$ & $\begin{array}{l}- \\
-\end{array}$ & & $\begin{array}{l}- \\
-\end{array}$ & \\
\hline Length (m) & - & & $L_{\text {pipe }}$ & 0.230 & $L_{\mathrm{RSH}}$ & 0.070 & $L_{\mathrm{PH}}$ & 0.0443 \\
\hline Width (m) & - & & & & $w_{\mathrm{RSH}}$ & 0.045 & $w_{\mathrm{PH}}$ & 0.0224 \\
\hline $\begin{array}{l}\text { Thickness } \\
(\mathrm{m})\end{array}$ & $t_{\mathrm{tip}}$ & 0.025 & & & $t_{\mathrm{RSH}}$ & 0.003 & $t_{\mathrm{PH}}$ & 0.00046 \\
\hline $\begin{array}{l}\text { Density } \\
\left(\mathrm{kg} / \mathrm{m}^{3}\right)\end{array}$ & $\rho_{\text {tip }}$ & 1810 & $\rho_{\text {pipe }}$ & 1810 & $\rho_{\mathrm{RSH}}$ & 1810 & $\rho_{\mathrm{PH}}$ & 7500 \\
\hline $\begin{array}{l}\text { Young's } \\
\text { modulus } \\
(\mathrm{GPa})\end{array}$ & $E_{\text {tip }}$ & 69 & $E_{\text {pipe }}$ & 75 & $E_{\mathrm{RSH}}$ & 75 & $E_{\mathrm{PH}}$ & 50 \\
\hline $\begin{array}{l}\text { Poisson } \\
\text { ratio }\end{array}$ & - & 0.33 & - & 0.3 & - & 0.3 & - & 0.33 \\
\hline $\begin{array}{l}\text { Piezoelectric } \\
\text { strain } \\
\text { constant } \\
(\mathrm{m} / \mathrm{V})\end{array}$ & - & - & - & - & - & - & $d_{31}$ & $\begin{array}{l}-150 \mathrm{e}- \\
12\end{array}$ \\
\hline $\begin{array}{l}\text { piezoelectric } \\
\text { stress/charge } \\
\text { constant } \\
\left(\mathrm{C} / \mathrm{m}^{2}\right)\end{array}$ & - & - & - & - & - & - & $d_{33}$ & 23.3 \\
\hline
\end{tabular}

A single arm of the UAV frame with an integrated harvester is excited to vibration by force $F_{0}$ obtained from the supply system containing a BLDC motor and a propeller. Both elements are located on the free end. The piezo harvester of the length of $L_{\mathrm{PH}}$, the width of $w_{\mathrm{PH}}$, and the thickness of $t_{\mathrm{PH}}$, integrated to the structure at the position $\left(x_{\mathrm{PH} 1}, x_{\mathrm{PH} 2}\right)$, was used to measure voltage from vibrations of the structure. 
Then, the general equation of transverse vibration of such an excited 1D mechanical structure with an integral harvester has the following form [12]:

$$
\begin{aligned}
& {\left[E_{\text {pipe }} I_{\text {pipe }}+E_{\mathrm{PH}} I_{\mathrm{PH}}\left(H\left(x-x_{\mathrm{PH} 2}\right)-H\left(x-x_{\mathrm{PH} 1}\right)\right)\right] \frac{\partial^{4} w(x, t)}{\partial x^{4}}} \\
& \quad+\left[m_{\mathrm{pipe}}+m_{\mathrm{PH}}\left(H\left(x-x_{\mathrm{PH} 2}\right)-H\left(x-x_{\mathrm{PH} 1}\right)\right)\right] \frac{\partial^{2} w(x, t)}{d t^{2}} \\
& \quad+-\Gamma V(t)\left[\frac{d \partial\left(x-x_{\mathrm{PH} 2}\right)}{d x}-\frac{d \partial\left(x-x_{\mathrm{PH} 1}\right)}{d x}\right]=F_{0} \delta\left(x-x_{0}\right)
\end{aligned}
$$

where $E_{\text {pipe }}$ - the Young modulus of the pipe, $E_{\mathrm{PH}}$ - the Young modulus of the piezo-harvester element, $I_{\text {pipe }}$ - the inertia moment of the pipe wherein $I_{\text {pipe }}=$ $\left[\pi\left(R_{\mathrm{out}}^{4}-R_{\mathrm{in}}^{4}\right)\right] / 4, I_{\mathrm{PH}}-$ the inertia moment of the piezo-harvester, $m_{\mathrm{pipe}}-$ the mass per unit length of the piezo pipe, $m_{\mathrm{PH}}$ - the mass per unit length of the piezoharvester, $H(x)$ - the Heaviside function, $\delta(x)$ - Dirac function along the $X$ axis, $V(t)$ - voltage flowing through the external resistive load $R, F_{0}$ - the force obtained from the supply system containing a BLDC motor and a propeller, $x_{\mathrm{PH} 1}-$ the begin location of the piezo-patch harvester on the host structure, $x_{\mathrm{PH} 2}$ - the ending location of the piezo-patch harvester on the host structure, $\Gamma$ - electromechanical coupling term.

The modal value of electromechanical coupling term $\tilde{\Gamma}_{n}$ in equation (1) of the system is expressed as:

$$
\tilde{\Gamma}_{n}=-E_{\mathrm{PH}} d_{31} w_{\mathrm{PH}} t_{c}
$$

where $t_{\mathrm{c}}$ - the distance between neutral axes of the tube and the piezo-harvester, $t_{c}=R_{C}+\frac{R_{\mathrm{out}}-R_{\mathrm{in}}}{2}+t_{\mathrm{ove}}+\frac{t_{\mathrm{PH}}}{2}, d_{33}-$ piezoelectric strain coefficient, $w_{\mathrm{PH}}-$ the width of the piezo harvester, $R_{C}$ - the radius of the tube to natural axis, $R_{\text {out }}$ - the outer radius of the tube, $R_{\text {in }}$ - the inner radius of the tube, $t_{\text {ove }}$ - the thickness of the overlay, $t_{\mathrm{PH}}-$ the thickness of the piero-harvester.

The piezoelectric harvester integrated with the host structure requires considering also the approach from the electrical point of view. For this purpose the electrical charge accumulated at its electrodes can be calculated over the whole surface area in the following form [12]:

$$
Q=\int_{x_{-} \mathrm{PH} 1}^{x_{-} \mathrm{PH} 2}\left(d_{31} E_{\mathrm{PH}} \bar{\delta}_{\mathrm{PH}}+\varepsilon_{33} E_{3}\right) w_{\mathrm{PH}} d x
$$

where $\varepsilon_{33}$ - permittivity at constant stress, $\bar{\delta}_{\mathrm{PH}}-$ bending strain along the middle surface of the piezolayer, $E_{3}$ - electric field.

Next, applying the Ohm's law, the current flowing through the load resistor $R$ can be expressed as:

$$
i(t)=\frac{V(t)}{R}=\frac{d Q}{d t}=\frac{d}{d t}\left[\int_{x_{-} \mathrm{PH} 1}^{x_{-} \mathrm{PH} 2}\left(d_{31} E_{\mathrm{PH}} \bar{\delta}_{\mathrm{PH}}+\varepsilon_{33} E_{3}\right) w_{\mathrm{PH}} d x\right]
$$

where $R$ - resistive load applied to the system.

Taking into account equation (4), it can be seen that the current $i(t)$ is strongly associated to strains of the piezoelectric harvester and the electrical field applied to its electrodes. This caused that the electrical circuit of the system can be obtained by substituting the electric field $E_{3}=-V(t) / t_{\mathrm{PH}}$ and the strain $\bar{\delta}_{\mathrm{PH}}=-t_{c} \frac{\partial^{2} w}{\partial x^{2}}$ in 
the following form [13]:

$$
C_{p} \frac{d V(t)}{d t}+\frac{V(t)}{R}+\Gamma\left[\int_{x_{-} \mathrm{PH} 1}^{x_{-} \mathrm{PH} 2} \frac{\partial^{3} w(x, t)}{\partial x^{2} \partial t} d x\right]=0
$$

where $C_{p}$ - capacitance of the piezo-patch harvester $C_{p}=\frac{\varepsilon_{33} w_{\mathrm{PH}} l_{\mathrm{PH}}}{t_{\mathrm{PH}}}$.

Both equations (1) and (5), refer to distributed electro-elastic model parameters of the piezo-patch harvester integrated to a $1 \mathrm{D}$ mechanical structure in physical coordinates. However, from the energy harvesting point of view, it should be analyzed in modal coordinates. For this purpose, vertical displacement of the beam as a host structure is represented as multiplication of an absolutely and uniformly convergent series of the eigenfunctions in the following form:

$$
w(x, t)=\sum_{n=1}^{\infty} \phi_{n}(x) \eta_{n}(t)
$$

where $\phi_{n}(x)$ - the mass normalized eigenfunction (mode shapes), $\eta_{n}(t)$ - the modal time response of the system for $n$th mode.

The considered structure, according to Figure 2, represents the cantilever beam. This caused that eigenvectors of this beam, after considering the boundary coordinates, given in equation (7), and split by geometric and time variables, can be expressed as [16]:

$$
\begin{aligned}
& w(0, t)=0,\left.\frac{\partial w(x, t)}{\partial x}\right|_{x=0}=0,\left(E_{\mathrm{pipe}} I_{\text {pipe }}+E_{\mathrm{PH}} I_{\mathrm{PH}}\left(H\left(x-x_{\mathrm{PH} 2}\right)\right.\right. \\
& -\left(H\left(x-x_{\mathrm{PH} 1}\right)\right) \frac{\partial^{2} w(x, t)}{\partial x^{2}}=\left.I_{\text {tip }} \frac{\partial^{3} w(x, t)}{\partial x \partial t^{2}}\right|_{x=L_{\text {_pipe }}} \text { and } \\
& \left(E_{\text {pipe }} I_{\text {pipe }}+E_{\mathrm{PH}} I_{\mathrm{PH}}\left(H\left(x-x_{\mathrm{PH} 2}\right)-\left(H\left(x-x_{\mathrm{PH} 1}\right)\right) \frac{\partial^{3} w(x, t)}{\partial x^{3}}\right.\right. \\
& =\left.m_{\mathrm{tip}} \frac{\partial^{2} w(x, t)}{\partial t^{2}}\right|_{x=L_{\text {_pipe }}} \\
& \phi_{n}(x)=\sin \left(\frac{\lambda_{n}}{L_{\text {pipe }}}\right) x-\sinh \left(\frac{\lambda_{n}}{L_{\text {pipe }}}\right) x+\beta\left[\cos \left(\frac{\lambda_{n}}{L_{\text {pipe }}}\right) x-\cosh \left(\frac{\lambda_{n}}{L_{\text {pipe }}}\right) x\right]
\end{aligned}
$$

where $\beta=\frac{-\cos \lambda_{n}-\cosh \lambda_{n}+\frac{\lambda_{n} m_{\text {tip }}}{m_{\text {pipe }} L_{\text {pipe }}}\left(\sin \lambda_{n}-\sinh \lambda_{n}\right)}{-\sin \lambda_{n}+\sinh \lambda_{n}-\frac{\lambda_{n} m_{\text {tip }}}{m_{\text {pipe }} L_{\text {pipe }}}\left(\cos \lambda_{n}-\cosh \lambda_{n}\right)} \quad$ for $n=1,2,3, \lambda_{n}$ - frequency parameter of an undamped host structure.

The obtained equation (8) put into equation (1) leads to solving the eigenvalue problem of the smart beam for short circuit conditions. Then, the natural frequency $\omega_{n}$ of the structure without the presence of the piezoelectric patch for $n$th mode can be presented in the following form:

$$
\omega_{n}=\lambda_{n}^{2} \sqrt{\frac{E_{\text {pipe }} I_{\text {pipe }}}{m_{\text {pipe }} L_{\text {pipe }}^{4}}}
$$

where $L_{\text {pipe }}$ - the length of the beam. 
Taking into account the modal analysis procedure of the 1D structure with a piezo harvester adjusted to its surface by using support, an electromechanical coupled ordinary differential equation for the modal time response $\eta_{n}$ can be expressed as [12]:

$$
\frac{d^{2} \eta_{n}(t)}{d t^{2}}+2 \xi_{n} \omega_{n} \frac{d \eta_{n}(t)}{d t}+\omega_{n}^{2} \eta_{n}(t)+\tilde{\Gamma}_{n} V(t)=f_{n}(t) \delta\left(x-x_{0}\right)
$$

where $\xi_{n}$ - modal damping ratio, $f_{n}(t)$ - modal force applied to the structure.

As a result, the modal electromechanical coupling term $\tilde{\Gamma}_{n}$ can be presented as:

$$
\tilde{\Gamma}_{n}=-E_{\mathrm{PH}} d_{31} w_{\mathrm{PH}} t_{c} \int_{x_{\_} \mathrm{PH} 1}^{x_{\_} \mathrm{PH} 2} \frac{d^{2} \phi_{n}(x)}{d x^{2}} d x=-\left.E_{\mathrm{PH}} d_{31} w_{\mathrm{PH}} t_{c} \frac{d \phi_{n}(x)}{d x}\right|_{x_{-} \mathrm{PH} 1} ^{x_{-} \mathrm{PH} 2} .
$$

The obtained modal electromechanical coupling term given by equation (11) and the vertical deflection from equation (8) put into equation (5) lead to modifying the electrical circuit equation in the following form:

$$
C_{p} \frac{d V(t)}{d t}+\frac{V(t)}{R}-\sum_{n=1}^{\infty} \tilde{\Gamma}_{n} \frac{d \eta_{n}(t)}{d t}=0
$$

The performed considerations of the cantilever beam with an integrated piezoharvester for modal coordinates allow to calculate the value of rectified voltage response accumulated on the resistor $R$ in the steady-state. For this purpose, assuming the harmonic form of the force generated from the system connected with the motor BLDC and the propeller, formed as $f(t)=F_{0} \sin (\omega t)=F_{0} e^{j \omega t}$, the modal voltage response $V(t)$ and the modal response of the system $\eta_{n}$ can also expressed in the following forms:

$$
V(t)=\operatorname{Re}\left\{\tilde{V}_{n} e^{j \omega t}\right\}, \eta_{n}=\operatorname{Re}\left\{\tilde{\eta}_{n} e^{j \omega t}\right\}
$$

Substituting equation (13) into equation (10) and equation (12) leads to modifyingthe electromechanical model of the electrical circuit system to the following form:

$$
\begin{gathered}
-\omega^{2} \tilde{\eta}_{n} e^{j \omega t}+2 j \xi_{n} \omega_{n} \omega \tilde{\eta}_{n} e^{j \omega t}+\omega_{n}^{2} \tilde{\eta}_{n} e^{j \omega t}-\tilde{\Gamma}_{n} V(t) e^{j \omega t}=F_{0} e^{j \omega t} \phi_{n}(x) \\
C_{p} V(t) j \omega e^{j \omega t}+\frac{V(t) e^{j \omega t}}{R}+e^{j \omega t} \sum_{n=1}^{\infty} j \tilde{\Gamma}_{n} \omega \tilde{\eta}_{n}=0
\end{gathered}
$$

Next, as a results of rejection the harmonic part $e^{j \omega t}$ from equation (14), the modal amplitude of the piezo-harvester response can be given by:

$$
\tilde{\eta}_{n}=\frac{\tilde{F}_{n}-\tilde{\Gamma}_{n} \tilde{V}}{\omega_{n}^{2}+2 j \xi_{n} \omega_{n} \omega-\omega^{2}}
$$

where $\tilde{F}_{n}$ - the modal force amplitude, wherein $\tilde{F}_{n}=F_{0} \phi_{n}\left(x_{0}\right), \tilde{V}$ - the modal voltage amplitude. 


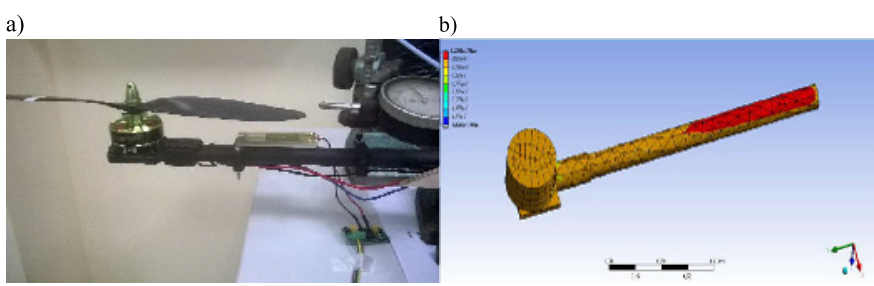

Fig. 3. (a) A single arm of a UAV system with a BLDC rotor, (b) a numerical model of the arm with tip mass.

Finally, the frequency response function of the modal voltage amplitude $\tilde{V}$ of the smart beam in the frequency domain can be express in the following form in the case of substituting equation (16) into equation (15) [13]:

$$
\tilde{V}(\omega)=\frac{\sum_{n=1}^{\infty} \frac{-j m_{\mathrm{PH}} \omega \tilde{\Gamma}_{n} \int_{x_{\mathrm{PH} \_1}}^{x_{\mathrm{PH} \_2}} \phi_{n}(x) d x}{\omega_{n}^{2}+2 j \xi_{n} \omega_{n} \omega-\omega^{2}}}{j \omega C_{p}+\frac{1}{R}+\sum_{n=1}^{\infty} \frac{j \omega \tilde{\Gamma}_{n}^{2}}{\omega_{n}^{2}+2 j \xi_{n} \omega_{n} \omega-\omega^{2}}} .
$$

\section{Numerical analysis of a single smart arm of the UAV with a BLDC rotor}

In this section the numerical investigations of a smart single arm of the UAV with a BLDC rotor, electrically powered from a battery shown in Figure 3a, are described. In order to design the vibration-based energy harvesting system, the eigenvalue problem and the location of the piezo-harvester on the structure is solved by FEM package. Taking into account analytical considerations described in the previous section, single UAV arm with a motor stand housing and the motor was modelled as a cantilever beam with tip mass by using Ansys Workbench software. It allows to perform the harmonic analysis of the structure and determine the strains of the single UAV arm that appear on the structure as a result of acting of different forces generated from abrushless motor with a propeller. In order to do it, the carbon-fiber tube of the length of $230 \mathrm{~mm}$ and a rectangular motor stand housing applied to the tube of the size given in Table 1 was modelled as a cantilever beam, while the BLDC rotor with an aluminum housing as a tip mass. Then, the obtained numerical model is shown in Figure 3b.

As it was mentioned before, the numerical investigations of the single UAV arm with the BLDC motor is carried out in a number of steps. In the first one, the eigenvalue problem of such a modelled structure with tip mass equalling $95 \mathrm{~g}$ (mass of the BLDC motor) is solved by using a harmonic response toolbox of the Ansys software. For this purpose, the range of frequency during the analysis was set between 10 and $700 \mathrm{~Hz}$, where it is possible to obtain a few of the first natural frequencies of the considered UAV arm. As a result, the frequency response function of the structure is calculated and shown in Figure 4.

The obtained FRF plot showed only the first two natural frequencies, $64.3 \mathrm{~Hz}$ and $678.2 \mathrm{~Hz}$, which are located at a large distance from each other. Taking into accountthe value of the $K_{v}$ rating parameter of the BLDC motor located on the platform in Figure $1\left(K_{v}=690 \mathrm{rpm} / \mathrm{V}\right)$ and the voltage from the battery $(14.7 \mathrm{~V})$, it 


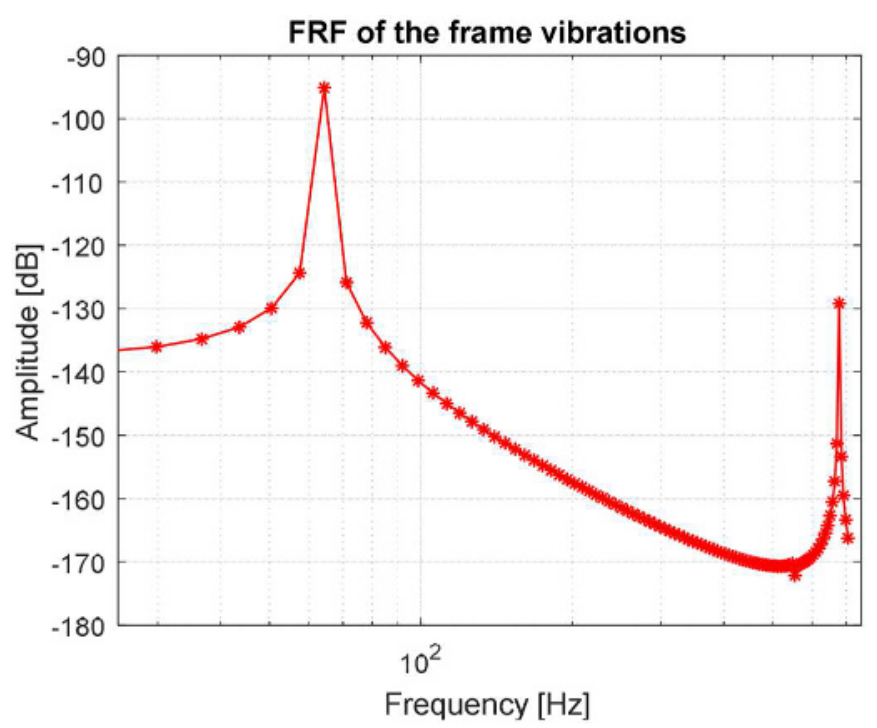

Fig. 4. Frequency response function of the copter arm with the tip mass of $95 \mathrm{~g}$.

can be assumed that the maximum working frequency of the considered system does not exceed $172 \mathrm{~Hz}$. As a result, the investigated system can work in the frequency range containing only the first natural frequency.

In the next step, the strains of the tube and its vertical deflections are numerically analysed. In this way, the quasi optimal location of the piezo-harvester element on the structure to obtain the maximum voltage from vibrations was assessed, similarly as it was done in reference [15]. For this purpose, firstly the excitation force that represents a thrust force needs to be defined. Taking into account specification of a brushless dc motor used in the system, it is known that its value of the thrust force depends on the frequency of the PWM signal. As a result a sinusoidal excitation force in the form $f(t)=0.02 \sin (2 \pi f t)$ was applied to the tip mass in the vertical direction, which is shown in Figure 5.

The numerical calculations are carried out for the assumed parameters: constant value of the supply voltage obtained from the battery $(14.7 \mathrm{~V})$ and different values of the PWM duty signal changes within the range from $20 \%$ up to $50 \%$ with the step of $10 \%$. This leads to obtaining four different sinusoidal signal excitations corresponding with frequencies of $31.7 \mathrm{~Hz}, 57.5 \mathrm{~Hz}, 66.1 \mathrm{~Hz}$ and $78.5 \mathrm{~Hz}$ and one signal of the frequency of $44 \mathrm{~Hz}-35 \%$ PWM duty signal. For the sinusoidal forces applied to the FEM model, the numerical investigations using a transient response toolbox of the Ansys software are performed and the results from this analysis are shown in Figures 6 and 7 , respectively.

The obtained results of bending strains and vertical deflection of the cantilever tube showed a significant increase of amplitudes especially in the case of excitation of the system with the frequency of $f=66.1 \mathrm{~Hz}$, which is close to the first natural frequency. Then, values of bending strains and the vertical deflections are significantly higher than in other cases. Taking into account Figure 6, it can be assumed that the best location of the piezo-stripe harvester on the tube is close to the fixed end, because the increase of the bending strains between particular nodes is biggest. In the case of the vertical deflection analysis, it can be seen that the best placement to locate the displacement sensor is vicinity of the rotor that it was located on the free end of the tube. However, taking into account the size of the propeller installed on the BLDC rotor, the supposed location should be shifted towards the centre of the tube. 


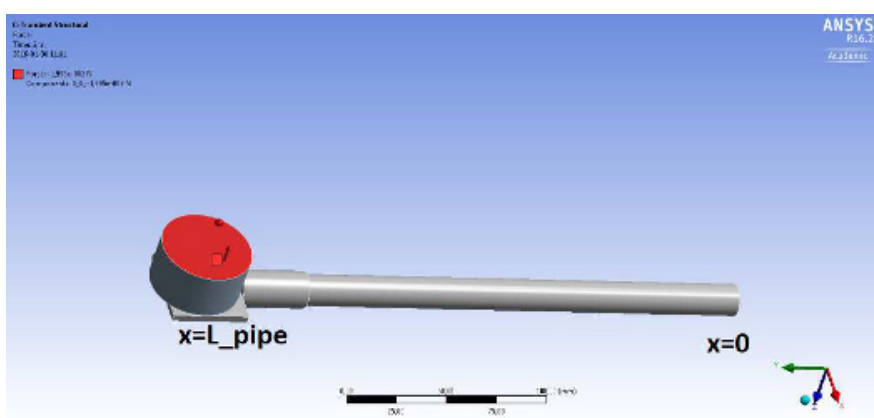

Fig. 5. The FEM model of a UAV single arm with a brushless DC motor and the applied sinusoidal force as a thrust force.
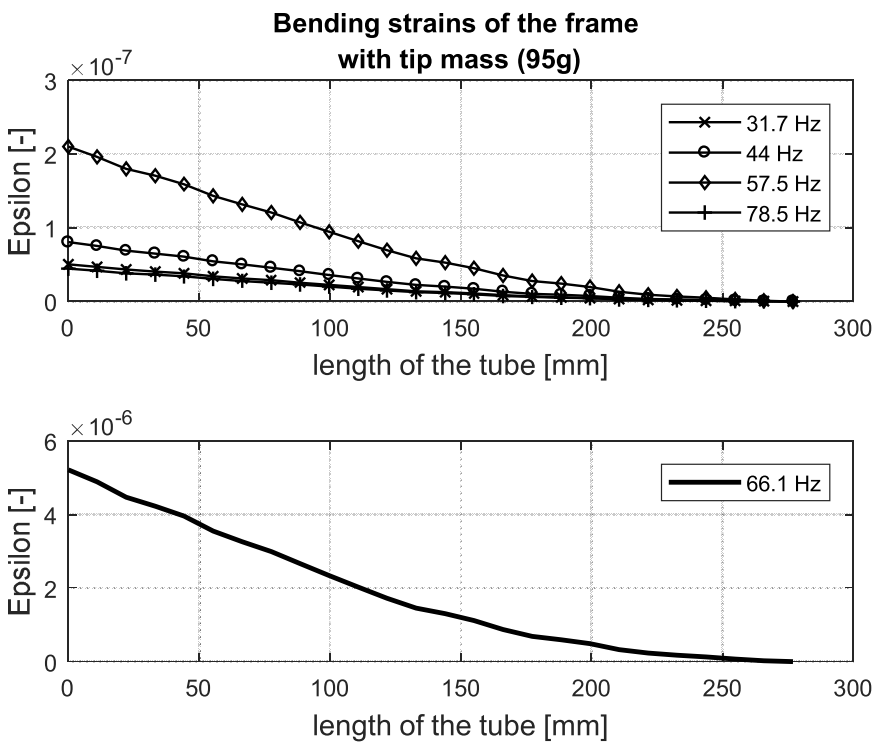

Fig. 6. The bending strains of the cantilever tube with the tip mass for different sinusoidal signal excitations applied to the tip mass.

The indicated piezo harvester location on a single arm of the UAV frame allows to calculate the amount of electrical power generated by the piezo-harvester that is a function of the excitation force applied to the system in the frequency range of $30-170 \mathrm{~Hz}$. For this purpose, firstly we need to assume a constant value of the resistive load connected to the system $R=100 \mathrm{k} \Omega$ and then - calculate the values of the capacitor $C_{p}$ attached to the piezo-harvester and the electromechanical coupling factor $\tilde{\Gamma}_{n}$ based on equations (6) and (11), respectively. Then, taking into account the square of the modulus of the obtained complex amplitude of the voltage from equation (16), additionally divided by resistive load $R$, we can obtain the power of the considered voltage.

The obtained power peak of the voltage generated by the piezo-harvester in Figure 8 indicate that the best vibration-based energy harvesting system parameters can be achieved for $40 \%$ duty cycle of the PWM signal applied to the BLDC motor. Then, the excitation frequency is close to the first natural frequency of the considered beam $(66.1 \mathrm{~Hz})$. In other cases, the values of the generated power are significantly lower, so they can be omitted in further analysis of the energy harvesting system. 

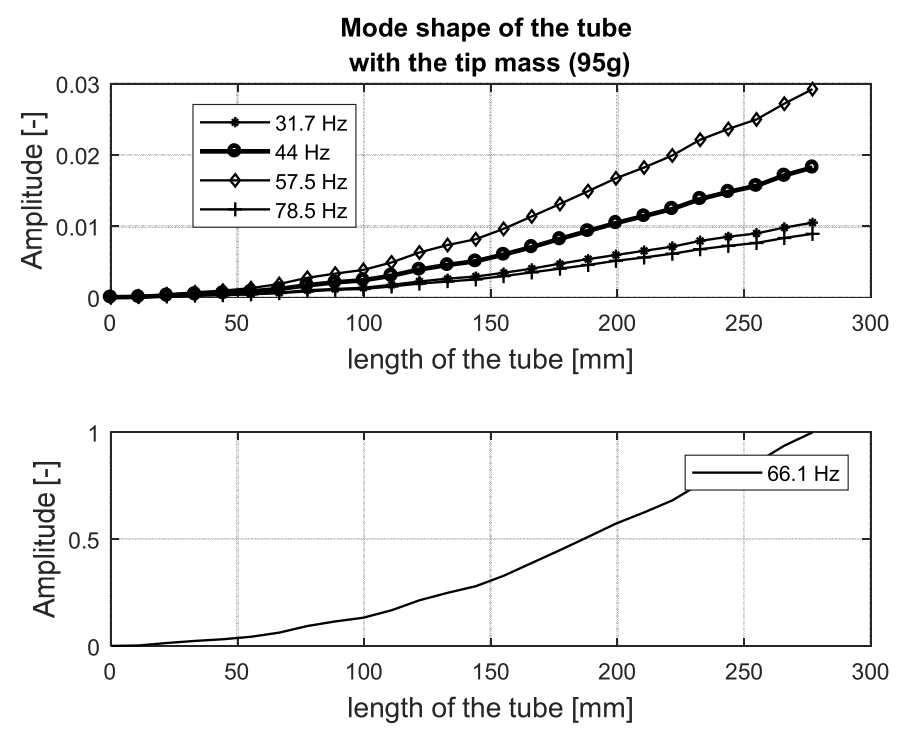

Fig. 7. The normalized mode shapes of the considered cantilever arm tube for different sinusoidal signal excitations applied to the tip mass.

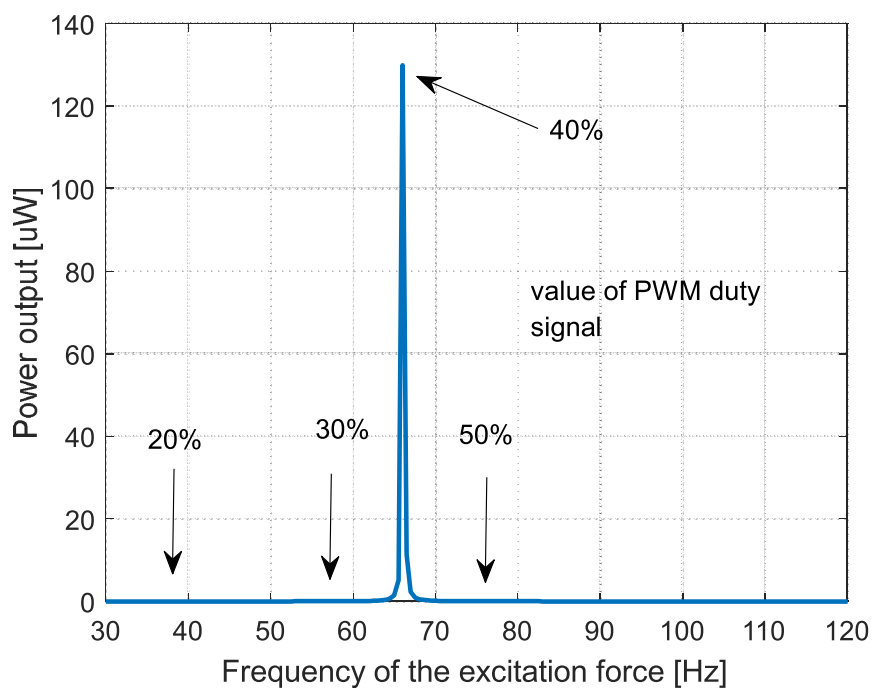

Fig. 8. The power of the voltage generated by the piezo-harvester located on the smart beam in the distance of $0-44.62 \mathrm{~mm}$ from fixed end for the first natural frequency of the beam $\omega_{1}$ versus the frequency of the excitation force generated by the tip mass.

\section{Experimental set-up and testing of energy harvesting system}

In this section, the performance of the energy harvesting system is tested on a laboratory stand. In order to do it, one end of a carbon fiber single arm of the UAV frame is fixed to the handle, while the free end is filled by a BLDC rotor 4822-690, developed by Mulistar company, and a propeller of the size of $12 \times 3.8$. The passive mechanical structurehas been additionally equipped with a piezo-harvester sensor PPA-1001 
a)

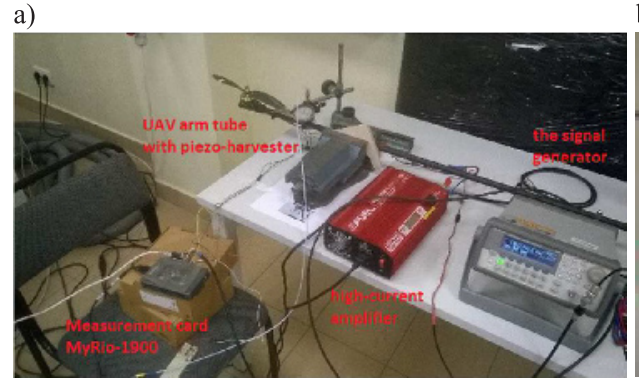

b)

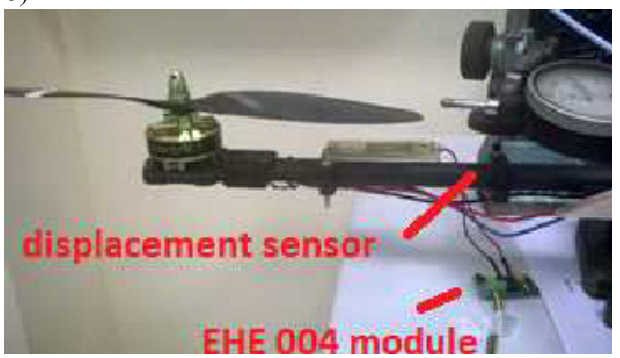

Fig. 9. (a) Photo of the laboratory stand, (b) photo of a single arm UAV with a displacement (eddy-current) sensor, a piezo-patch harvester PPA-1001 and the conditioning system EHE004.

located very close to the fixed end of the arm tube. Moreover, the energy harvesting conditioning system EHE 004 is used to measure the voltage from the capacitor.

Taking into account the requirements of the BLDC rotor and the energy harvesting system, the laboratory stand is also retrofitted in a signal generator developed by Agilent, a high-current amplifier and an eddy-current sensor that allows to measure vibrations of the investigated structure. In order to record both the excitation and the measurement signals, the lab stand was additionally equipped with a measurement card myRio 1900, developed by National Instruments, and a laptop with LabView software.

The vibration-based energy harvesting system is tested in a number of steps. In the first one, the structure is investigated in the time domain using Digital Signal Analyzer. For this purpose, the Pulse Width Modulation (PWM) signal was generated from a signal generator developed by Agilent. The frequency of the signal and its amplitude were set at $50 \mathrm{~Hz}$ and $5 \mathrm{~V}$, respectively, while the duty cycle of the PWM signal was changed in the range from $1 \mathrm{~ms}$ up to $2 \mathrm{~ms}$. It corresponds withgeneratingthe thrust force by the rotor in the range of $0-100 \%$. For such a prepared excitation signal, the vibrations of the structure are measured using the eddy-current sensor located over the frame in the distance of $80 \mathrm{~mm}$ from the fixed end and with a gap between the head of the sensor and the structure of $0.5 \mathrm{~mm}$.

The measurement signal of the vertical displacement of the structure shown in Figure 10 in order to verify the numerical results are transformed to the frequency domain. As a result of using the Fast Fourier Transform, the spectrum of the recorded vibration signal in the range of $4-12 \mathrm{~s}$ are determined. The highest obtained amplitude peak, as it can be seen in Figure 10b, is located in the first natural frequency of the investigated structure. It indicates that the numerical results are correct and the considered system should work in the frequency of about $66 \mathrm{~Hz}$. Finally, it allows to generate the maximum voltage from vibrations.

In the second step, the measurements are repeated for the same type of signal excitation. However, in this case, the spectrum of the amplitude vibrations are determined separately for each duty cycle of the PWM signal in the range of 1-1.5 ms because, as it turned out, further increase of the duty cycle on the lab-stand was very dangerous for the researcher. The obtained results, presented in Figure 11b, show a significant increase of the amplitude peak vibrations, especially in the case of the system excitation with the duty cycle that equals $1.4 \mathrm{~ms}$, corresponding to the first natural frequency. Other amplitude peaks, visible in Figure 11b, lower than 0.13 , present only excitation frequencies applied to the rotor.

Next, the tested system was investigated in order to assess its ability to generate voltage from a vibrating piezo-stripe harvester. For this purpose, similarly to the previous case, the PWM signal from the generator and the high-current amplifier was 
a)

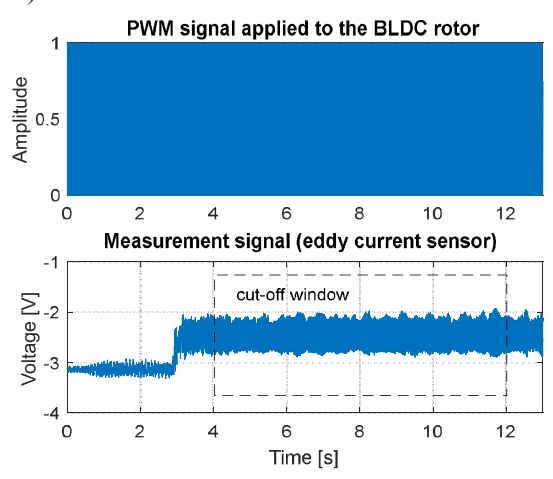

b)

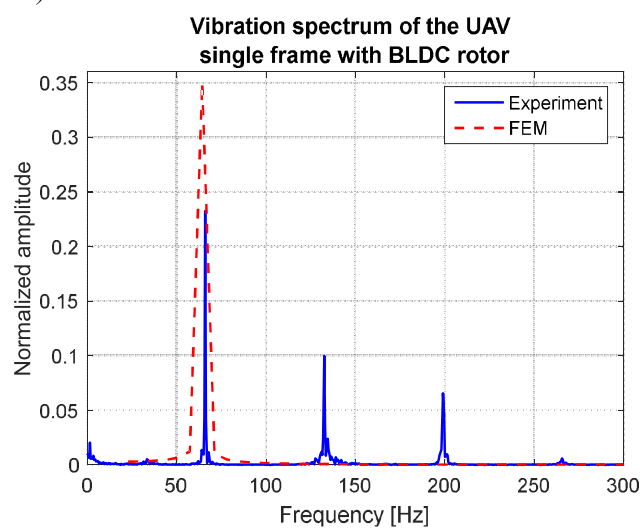

Fig. 10. (a) The recorded excitation and vibration signals of the energy harvesting system in the time domain, (b) the comparison of the vibration spectrum of the single frame of UAV and the numerical model.

a)
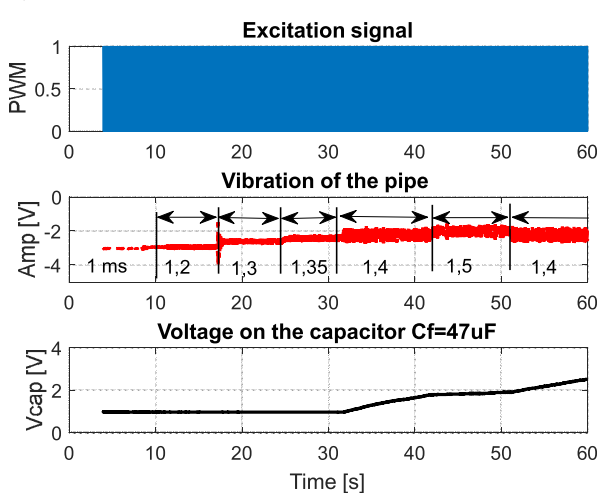

b)

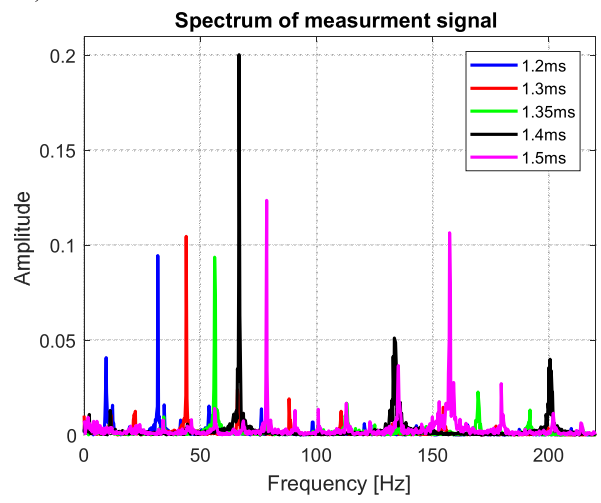

Fig. 11. (a) Recorded excitation and measurement signals during test, (b) spectrum of amplitude vibrations of the single UAV arm versus excitation force applied to the rotor.

applied to abrushless DC rotor with the duty cycle of $1-1.5 \mathrm{~ms}$. The measured and recorded voltage signal on the output capacitor $\left(V_{\mathrm{CAP}}\right)$ showed a very fast increase of its value for the excitation signal with the duty cycle of $1.4 \mathrm{~ms}$, corresponding with the closed-resonance frequency $(66 \mathrm{~Hz})$. In other cases, as it can be seen in Figure 11a, the process of charging the capacitor is slowed down and the voltage on its signal has a constant value.

The next step of the experimental set-up was associated with assessing the effectiveness of the energy harvesting systems which can be installed on the board of UAV platforms. For this purpose, the investigations are carried out in two steps. In the first one, the conditioning module EHE004 was set to generate the voltage output signal only of $3.7 \mathrm{~V}$ up to $5 \mathrm{~V}$. On the other hand, in the next step, the boundary value of the resistive load $R$, connected to the system, was chosen in order to obtain only constant value of the output voltage $V_{\text {out }}$ from the system.

As it can be seen in Figure 12, the system was loaded by the resistive load $R$ of the value of $100 \mathrm{k} \Omega$ and $140 \mathrm{k} \Omega$. Taking into account a higher resistive load, it can be noticed that the energy harvester system gave better results, because the 
a)

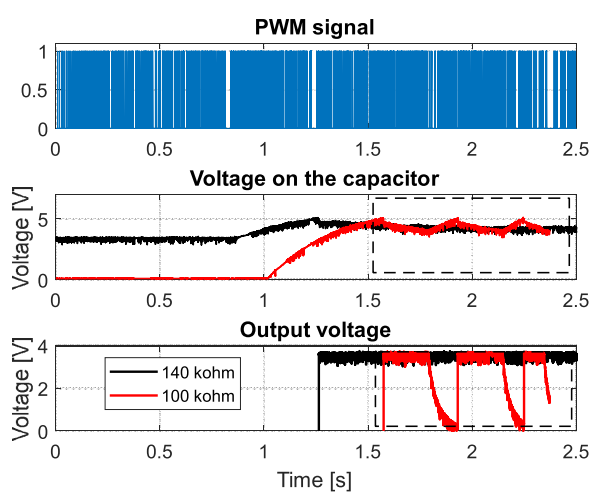

b)

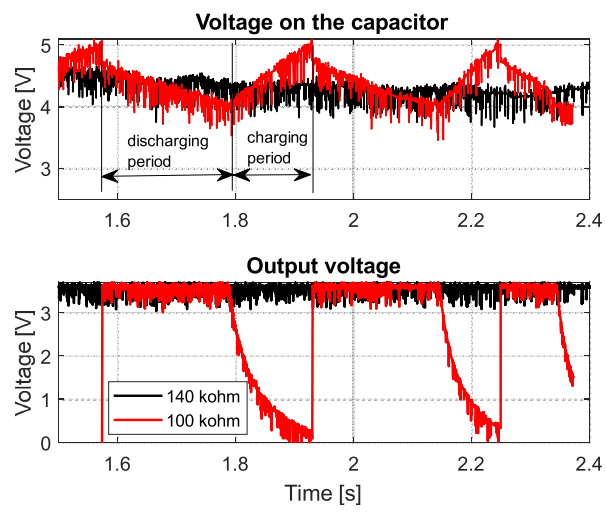

Fig. 12. The comparison of the output signal $V_{\text {out }}$ and the voltage $V_{\mathrm{CAP}}$ accumulated on the capacitor located on the conditioning system EHE004.

obtained voltage output was kept on the level of $3.7 \mathrm{~V}$. In the case of a lower value of the resistive loads than $140 \mathrm{k} \Omega$, the system generates only a periodical output signal whose duration time depends on its value. The obtained behavior of the system from the electrical point of view is only associated to the process of charging and discharging the output capacitor of the module EHE004.

The obtained experimental results allow to estimate the root mean square value for the output voltages achieved from the measurements according to equation (18), and once again verify the numerical results. The calculations of the peak voltage outputs are carried out in two steps. In the first one, the peaks are determined for different PWM signal applied to the BLDC rotor, while in the second step - for a chosen PWM signal but different resistive load $R$. In both cases all peaks are calculated for a constant value of the amount of the voltage signal samples $(N=13333)$

$$
\mathrm{RMS}_{V_{\text {_out }}}=\sqrt{\frac{1}{N} \sum_{i=1}^{N} V_{\text {out }}^{2}} .
$$

Taking into account Figure 13a, it can noticed that the obtained results properly verify the numerical results shown in Figure 8. In both cases the duty cycle of the excitation signal significantly influences the peaks of the voltage outputs. In the considered cases the highest peak is achieved for $40 \%$ of the duty cycle, but the lowest one - for the duty cycle lower than $40 \%$. As a result, it can be noted that the maximum values of the root square mean value of the output voltage signal are achieved in the vicinity of the first natural frequency of the considered system. Similar results are obtained in the case of the resistive load analysis connected to the system. As it can be seen in Figure 13b, the highest efficiency of the energy harvesting system is obtained for $40 \%$ of the duty cycle of the PWM and the resistive load equals $140 \mathrm{k} \Omega$. Then, the electric current flowingthrough the system has a fixed value equals $\left(I_{\text {out }}=0.26 \mu \mathrm{A}\right)$.

The generated voltage from the piezo-harvester and the electric current flowing through resistor allow to verify numerical results shown in Figure 8. For this purpose taking into account parameters of input power of the system dedicated to power supply of the copter $\left(V_{\text {in }}=14.7 \mathrm{~V}, I_{\text {in }}=4 \mathrm{~A}\right.$ for $40 \%$ of the duty cycle of the PWM signal applied to BLDC rotor with propeller) and output power can calculate efficiency of 
a)

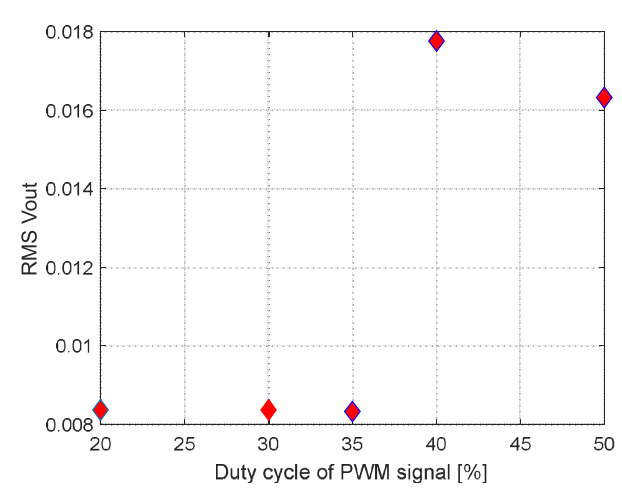

b)

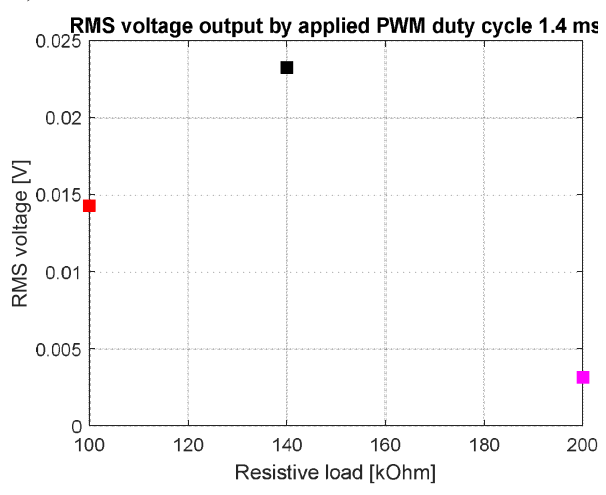

Fig. 13. The root mean square value of voltage signals from the piezo-harvester PPA-1001 located on the smart arm of a six-rotor UAV versus (a) different values of the PWM duty applied to a BLDC rotor, (b) different resistive load $R$.

the system in the following form:

$$
\eta_{\mathrm{ele}}=\frac{U_{\mathrm{out}} \cdot I_{\mathrm{out}}}{U_{\mathrm{in}} \cdot I_{\mathrm{in}}} \cdot 100 \%=\frac{3.7 \mathrm{~V} \cdot 26 \mu \mathrm{A}}{14.7 \mathrm{~V} \cdot 4 \mathrm{~A}} \cdot 100 \%=0.000166
$$

The obtained value of efficiency of the energy-harvesting system is very low, because input power was set to whole energy power system of rotor. It caused that the proposed vibration-based energy harvesting can be used for further research only with the same piezo-harvester elements located on each arm of the copter. Then, such obtain system perhaps allow to supply other devices located on the UAV platform.

\section{Summary and conclusions}

One-dimensional structures made of a beam with various boundary conditions and integrated piezo-patches stripes are commonly used in aerospace, biomedical and automotive applications to harvest energy from vibrations. The aforementioned fields of applications caused that such kinds of mechanical structures have been a subject of many articles in recent two decades. This caused that our investigations are focused on determining distributed parameters of a piezoelectric harvester located on a single arm of a UAV six-rotor and check the possibilities of the considered structure to harvest energy. For this purpose, as the first step, a modal analysis of the chosen structure is carried out using the finite element method. The obtained results, presented in Figure 4, show that only the first natural frequency $64.3 \mathrm{~Hz}$ can be taken into account for further analysis, because the maximum frequency generated by the BLDC motor does not exceed $172 \mathrm{~Hz}$. Further numerical investigations allow to determine the best location of the piezo-harvester on the structure in order to harvest the maximum energy amount from vibrations. For this purpose, the strain fields analysis of a single arm of the UAV frame is performed for different sinusoidal excitation forcesput atthe PWM duty of the signal applied to the rotor. The obtained results, presented in Figure 6, indicate that the best location of the piezo-harvester on the structure is close to the fixed end of the structure, and the largest values of the strains are achieved in the case of the system excitation of the frequency of $f=66.1 \mathrm{~Hz}$, which is close to first natural frequency. The determined harvester location on the structure and the 
calculated modal values of the electromechanical coupling factors allow to obtain the voltage $V$ for a mechanical model determined on the bases of analytical approaches. The obtained peak power of the voltage generated by the piezo-harvester, shown in Figure 8, indicate that the best vibration-based energy harvesting system parameters can be achieved for the system ay $40 \%$ of the PWM duty signal applied to the BLDC motor. Then, the excitation frequency is close to the first natural frequency of the considered beam $(66.1 \mathrm{~Hz})$. On the other hand, the values of the generated power from vibrations are significantly lower, so it can be omitted to analysis of the energy harvesting system.

Experimental investigations of the energy harvesting system carried out on the laboratory stand for real a structure properly verified the numerical results. In the first step, the highest amplitude peak of the beam displacement was obtained for $40 \%$ of the duty cycle of the PWM signal applied to the BLDC rotor. As a result, the frequency excitation of the structure to vibrations is close to the first natural frequency. In the next step, the recorded voltage from the piezo harvester (see Fig. 11a) indicate that the fastest increase of its value is achieved for the excitation signal of the same duty cycle (40\%). In other cases, the process of charging the capacitor is stopped and the voltage on its signal has a constant value. In the last step of the experimental investigations, the recorded voltage signals from the conditioning system allow to assess the effectiveness of the energy harvesting systems which can be installed on the board of UAV platforms. The obtained results, presented in Figures 12 and 13, show that the highest efficiency of the energy harvesting system is obtained for $40 \%$ of the duty cycle of PWM and the resistive load $R=140 \mathrm{k} \Omega$ connected to the system. Then, the electric current flowing through the system has a fixed value and it can be used to supply other devices located on the UAV platform. In other cases, the considered effectiveness of the system is very low because the system generates only a periodical output signal.

Summing up, the performed investigations of a piezoelectric harvester attached to a single arm of a six rotor UAV frame enabled exploring the harvesting performance of the system with reference to many copters. The obtained results indicate that it is possible to prolong the flight duration time of copters by using flexible batteries located on the UAV platform, but in the case of adding piezo-patch harvesters on each arm of the copter. This caused that further investigations will be carried out to obtain more energy harvested from piezo-harvesters located on each arm of a copter. As a result, the harvest energy system will be tested during indoor and outdoor flights.

This work has been supported by grant No. WZ/WM/1/2019 of Bialystok University of Technology, financed by Polish Ministry of Science and Higher Education.

Open Access This is an open access article distributed under the terms of the Creative Commons Attribution License (http://creativecommons.org/licenses/by/4.0), which permits unrestricted use, distribution, and reproduction in any medium, provided the original work is properly cited.

\section{References}

1. M.J. Nowlan, J.C. Maglitta, G. Darkazalli, T. Lamp, in Proceedings of the 26th Photovoltaic Specialists Conference, Anaheim, 1997, p. 1149

2. A. Koszewnik, K. Wernio, Acta Mech. Autom. 10, 4 (2016)

3. M. Krack, N. Aboulfotoh, J. Twiefel, J. Wallaschek, L.A. Bergman, A.F. Vakakis, Arch. Appl. Mech. 87, 4 (2017)

4. P. Wolszczak, K. Lygas, G. Litak, Mech. Syst. Signal Process. 107, 43 (2018) 
5. A. Koszewnik, P. Grzes, W. Walendziuk, Eur. Phys. J. Special Topics 224, 2719 (2015)

6. Z. Gosiewski, A. Koszewnik, Mech. Syst. Signal Process. 36, 136 (2013)

7. M. Nielsen-Lönn, P. Angelov, J.J. Wikner, A. Alvandpour, Analog. Integr. Circ. Signal Process 98, 441 (2019)

8. S.R. Anton, A. Erturk, D.J. Inman, Smart Mater. Struct. 19, 11 (2010)

9. S.R. Anton, Ph.D. thesis, Virginia Polytechnic Institute and State University, 2011

10. D. Oldziej, Z. Gosiewski, in Proceedings of Mechatronic Systems and Materials Conference, Poland 2012, edited by Z. Gosiewski, Z. Kulesza (Solid State Phenomena, Switzerland, 2013), p. 220

11. W. Walendziuk, D. Oldziej, P. Golinski, in Proceedings of WILGA Symposium on Photonics Applications and Web Engineering, A laboratory stand for research concerning drive units applied in unmanned flying micro vehicles, Poland, 2015, edited by R.S. Romaniuk (Warsaw University of Technology, Warsaw, 2015), p. 9662

12. A. Ertuk, D. Inman, J. Intell. Mater. Syst. Struct. 20, 5 (2009)

13. S. Rafique, Piezoelectric Vibration Energy Harvesting, Modeling and Experiments (Springer, Switzerland, 2018)

14. A. Preumont, Twelve Lectures on Structural Dynamics (Springer, New York, 2013)

15. A. Koszewnik, Z. Gosiewski, Eur. Phys. J. Plus 131, 232 (2016)

16. N.H. Diyana, G.A. Muthalif, M.N. Fakhzan, A.N Nordin, Procedia Eng. 41, 1228 (2012) 